\title{
Submillimeter observations of IRAS and WISE debris disk candidates ${ }^{\star}$
}

\author{
J. Bulger ${ }^{1}$, T. Hufford ${ }^{2}$, A. Schneider ${ }^{2}$, J. Patience ${ }^{1,3}$, I. Song ${ }^{2}$, R. J. De Rosa ${ }^{3}$, A. Rajan ${ }^{3}$, C. D. Dowell ${ }^{4}$, \\ D. McCarthy ${ }^{5}$, and C. Kulesa ${ }^{5}$
}

1 School of Physics, University of Exeter, Exeter, EX4 6LZ, UK e-mail: joanna@astro.ex.ac.uk

2 Department of Physics and Astronomy, The University of Georgia, Athens, GA 30602-2451, USA

3 School of Earth and Space Exploration, Arizona State University, Tempe, AZ 85281, USA

4 Department of Physics and Astronomy, Caltech, MC 249-17, Pasadena, CA 91125, USA

5 The Department of Astronomy and Steward Observatory, The University of Arizona, Tucson, AZ 85721, USA

Received 15 April 2013 / Accepted 10 July 2013

\section{ABSTRACT}

\begin{abstract}
A set of six debris disk candidates identified with IRAS or WISE excesses were observed at either $350 \mu \mathrm{m}$ or $450 \mu \mathrm{m}$ with the Caltech Submillimeter Observatory. Five of the targets - HIP 51658, HIP 68160, HIP 73512, HIP 76375, and HIP 112460 - have among the largest measured excess emission from cold dust from IRAS in the $25-100 \mu \mathrm{m}$ bands. Single temperature blackbody fits to the excess dust emission of these sources predict 350-450 $\mu$ m fluxes above $240 \mathrm{mJy}$. The final target - HIP 73165 - exhibits weak excess emission above the stellar photosphere from WISE measurements at $22 \mu \mathrm{m}$, indicative of a population of warm circumstellar dust. None of the six targets were detected, with $3 \sigma$ upper limits ranging from 51-239 mJy. These limits are significantly below the expected fluxes from SED fitting. Two potential causes of the null detections were explored - companion stars and contamination. To investigate the possible influence of companion stars, imaging data were analyzed from new adaptive optics data from the ARIES instrument on the 6.5 m MMT and archival HST, Gemini NIRI, and POSS/2MASS data. The images are sensitive to all stellar companions beyond a radius of 1-94 AU, with the inner limit depending on the distance and brightness of each target. One target is identified as a binary system, but with a separation too large to impact the disk. While the gravitational effects of a companion do not appear to provide an explanation for the submm upper limits, the majority of the IRAS excess targets show evidence for contaminating sources, based on investigation of higher resolution WISE and archival Spitzer and Herschel images. Finally, the exploratory submm measurements of the WISE excess source suggest that the hot dust present around these targets is not matched by a comparable population of colder, outer dust. More extensive and more sensitive Herschel observations of WISE excess sources will build upon this initial example to further define the characteristics of warm debris disks sources.
\end{abstract}

Key words. submillimeter: planetary systems - circumstellar matter - stars: imaging - techniques: high angular resolution

\section{Introduction}

Debris disks were first identified with the Infrared Astronomical Satellite (IRAS), from the observation that the far-IR flux of Vega (Aumann et al. 1984) was significantly brighter than expected from the stellar photosphere. Excess emission above the level of a stellar photosphere at wavelengths from the mid-IR to the millimeter provides evidence of reprocessed starlight emitted by a circumstellar debris disk of dust (see reviews by Zuckerman 2001; Wyatt 2008). The disk origin of these excesses has been confirmed with spatially resolved imaging (e.g. Smith \& Terrile 1984; Holland et al. 1998). Given the timescale for the dust to spiral into the star or be ejected from the system, these dust disks must be sustained by an ongoing process such as the collisional grinding of planetesimals into smaller particles (Backman \& Paresce 1993) or an event such as a catastrophic collision of planets (Cameron 1997).

While IRAS provided an all-sky survey for debris disks with a typical sensitivity of $\left(L_{\text {dust }} / L_{\star}\right) \sim 10^{-5}$, subsequent pointed observations from the Spitzer Space Telescope (Werner et al. 2004), achieved an order of magnitude greater sensitivity. The

\footnotetext{
* Herschel is an ESA space observatory with science instruments provided by European-led Principal Investigator consortia and with important participation from NASA.
}

combined results of the all-sky and pointed observations have identified over 200 debris disks among field stars (e.g. Mannings \& Barlow 1998; Moór et al. 2006; Rhee et al. 2007; Habing et al. 1999; Silverstone 2000; Rieke et al. 2005; Su et al. 2006; Bryden et al. 2006; Beichman et al. 2006; Hillenbrand et al. 2008; Carpenter et al. 2009). Assuming a single temperature blackbody model for the excess emission in the spectral energy distribution (SED), it is possible to classify debris disks as warm (equivalent to that of our Asteroid belt), with $T_{\text {dust }} \approx 100-250 \mathrm{~K}$, that lie at distances $\lesssim 10 \mathrm{AU}$, and cold debris disks (equivalent to that of our Kuiper belt), with $T_{\text {dust }} \lesssim 50 \mathrm{~K}$, typical distances of several hundred AU.

With the more recent Herschel and Wide-field Infrared Survey Explorer (WISE) missions, it is possible to conduct pointed searches for cold debris disks down to the Kuiper belt level $\left(L_{\text {dust }} / L_{\star}\right) \sim 10^{-7}$ to $10^{-6}$ (Stern 1996) and all-sky surveys for warm debris disks at a sensitivity level that was previously only obtained with Spitzer pointed observations. In addition to the sensitivity offered by both Herschel and WISE, the significant improvement of spatial resolution is important to identify possible sources of contamination. Examples of debris disks identified through IRAS excesses (e.g. Moór et al. 2006; Rhee et al. 2007) that are actually due to confusion from background source contamination have been revealed by higher resolution 
Table 1. Observed sample.

\begin{tabular}{|c|c|c|c|c|c|c|c|c|c|c|}
\hline Name & $\begin{array}{c}\mathrm{RA} \\
(\mathrm{J} 2000)\end{array}$ & $\begin{array}{c}\text { Dec } \\
(\mathrm{J} 2000)\end{array}$ & $\begin{array}{l}\text { Prop. mot. } \\
(\text { ("/yr) }\end{array}$ & $\begin{array}{c}D \\
(\mathrm{pc})\end{array}$ & SpTy & $\begin{array}{l}\text { Age } \\
(\mathrm{Myr})\end{array}$ & $\begin{array}{l}\text { Excess } \\
\text { source }\end{array}$ & $\begin{array}{c}T_{\text {dust }} \\
(\mathrm{K}) \\
\end{array}$ & $L_{\text {dust }} / L_{\star}$ & Ref. \\
\hline HIP 51658 & $10: 33: 13.88$ & $+40: 25: 31.65$ & $-0.142,0.008$ & $34.6 \pm 0.6$ & A7 & 200 & IRAS & 40 & $1.06 \times 10^{-4}$ & 1 \\
\hline HIP 68160 & $13: 57: 16.13$ & $+23: 21: 44.37$ & $-0.330,-0.158$ & $38 \pm 1$ & K0 & 230 & IRAS & & $1.49 \times 10^{-3}$ & 2 \\
\hline HIP 73165 & $14: 57: 11.01$ & $-04: 20: 47.32$ & $-0.097,-0.153$ & $26.9 \pm 0.2$ & F0 & & WISE & & & \\
\hline HIP 73512 & $15: 01: 29.96$ & $+15: 52: 08.45$ & $0.102,-0.238$ & $30 \pm 1$ & $\mathrm{~K} 2$ & $3000 ?$ & IRAS & 85 & $1.17 \times 10^{-3}$ & 1 \\
\hline HIP $76375^{a}$ & $15: 35: 56.61$ & $+40: 25: 31.65$ & $-0.448,0.051$ & $22 \pm 0.3$ & K3 & $5000 ?$ & IRAS & 29 & $7.86 \times 10^{-4}$ & 1 \\
\hline HIP 112460 & $22: 46: 49.81$ & $+44: 20: 03.10$ & $-0.705,-0.461$ & $5.12 \pm 0.05$ & M3.5 & 500 & IRAS & 19 & & 3 \\
\hline
\end{tabular}

Notes. ${ }^{(a)}$ Known binary reported in the literature.

References. (1) Rhee et al. (2007); (2) Moór et al. (2006); (3) Lestrade et al. (2006).

ground-based observations carried out in the IR and submm (e.g. Song et al. 2002; Jayawardhana et al. 2002; Lisse et al. 2002; Sheret et al. 2004).

Mid-IR to far-IR observations have been critical in identifying the current population of debris disks, however measurements at longer wavelengths in the submm/mm wavelength range provide the best means to estimate one of the most important properties of a disk, the dust mass. For disks that are spatially resolved in the submm, it is also possible to determine additional disk characteristics such as size and inclination which are degenerate with other disk parameters in an SED fit. For disks that have imaged asymmetries, these structures can encode the effects of gravitational interactions (e.g. Liou \& Zook 1999; Kuchner \& Holman 2003; Wyatt 2006; Quillen \& Faber 2006). The submm/mm wavelength range is ideal for studying the interaction of the disk and planets, i.e. in the case of the multiple planet, debris disk system around HR 8799 (e.g. Patience et al. 2011; Hughes et al. 2011), since the large grains are expected to remain in resonances with the planets, while the smaller grains evolve into axisymmetric structures due to scattering or radiation pressure (Wyatt 2006).

In this paper, we present the results of a submm and IR imaging study of several IRAS excess stars and one WISE excess star. This sample includes previously known systems with cold dust (Moór et al. 2006; Rhee et al. 2007) and newly identified debris disk candidates with evidence of either cold or warm dust. The properties of the sample are summarized in Sect. 2. The single-dish submm observations, infrared imaging and archival searches are described in Sect. 3. In Sect. 4, the data reduction and analysis of our submm and IR observations is presented. The results and implications of the IR image analysis is given in Sect. 5. In Sect. 6, we draw comparisons of our investigation to previous submm studies of debris disks. Finally, the conclusions of our study are given Sect. 7 .

\section{The sample}

The target sample for this investigation of cold and warm excess debris disks is drawn from two main sources - the IRAS Faint Source Catalog (FSC v.2) (Moshir et al. 1992) and results from the WISE All-Sky Data Release (Cutri \& et al. 2012). We selected a sample of cold excess disk candidates from the literature (Lestrade et al. 2006; Moór et al. 2006; Rhee et al. 2007) that satisfy three criteria: (1) a large IRAS excesses at $60 \mu \mathrm{m}$ or $100 \mu \mathrm{m}$; (2) a distance within $100 \mathrm{pc}$; and (3) a declination above $-30^{\circ}$. SEDs were constructed, and a single temperature blackbody model was fit to the IRAS excesses. Targets with an expected $350 \mu \mathrm{m}$ flux greater than $100 \mathrm{mJy}$ were considered for this study, and the SEDs of the targets are shown in Figs. 1 and 2.
Due to weather considerations, we focused our observations on sources with the greatest predicted $350 \mu \mathrm{m}$ flux and and with nearby distances $(<40 \mathrm{pc})$, in order to obtain meaningful limits and have the capacity to resolve the disks of the nearest systems. A total of 5 cold debris disks candidates were observed in this study, and are listed in Table 1, along with a summary of the modelled dust properties from the literature. The second type of target with evidence for warmer dust was drawn from the WISE $(3.4 \mu \mathrm{m}, 4.6 \mu \mathrm{m}, 12 \mu \mathrm{m}$, and $22 \mu \mathrm{m})$ All-Sky Data Release. The identification of a WISE W3 $(12 \mu \mathrm{m})$ and/or W4 (22 $\mu \mathrm{m})$ band excess was based on a careful cross-correlation of the WISE and HiPparcos catalogues (Perryman \& ESA 1997; van Leeuwen 2007) with a distance cut of 100 pc and an estimate of the photospheric flux from theoretical atmosphere models (Hauschildt et al. 1999). Due to the limited clear weather windows during the observing runs, it was only possible to observe one warm excess source - HIP 73615. HIP 73615 has an excess only in the WISE W4 band, and the excess above the photosphere is 34 times the uncertainty in the photometry. The properties of the target are listed in Table 1, and the SED for this warm debris disk candidate is plotted in Fig. 3.

\section{Observations}

\subsection{Submillimeter observations}

Observations of the six sources were made with the SHARCII bolometer array (Dowell et al. 2003) on the $10.4 \mathrm{~m}$ Caltech Submillimeter Observatory (CSO) on Mauna Kea. SHARCII contains a $12 \times 32$ array of pop-up bolometers with a $>90 \%$ filling factor over the field and provides background-limited $350 \mu \mathrm{m}$ and $450 \mu \mathrm{m}$ maps. For the closest systems, we obtained $350 \mu \mathrm{m}$ maps to potentially resolve the disks. For the remaining targets we measured $450 \mu \mathrm{m}$ maps to measure longer wavelength fluxes distinct from the Herschel bands. Table 2 which wavelengths were observed for each source and the exposure times. The field-of-view of the SHARCII maps was $\sim 2^{\prime} \times 00^{\prime} 6$, and the beam sizes at $350 \mu \mathrm{m}$ and $450 \mu \mathrm{m}$ are $\sim 88^{\prime \prime} 5$ and $\sim 10$. $^{\prime} 0$, respectively. The beam shape was stabilized by the active primary surface correction (Leong et al. 2006), which corrects for dish surface imperfections and changes in the gravity vector as a function of elevation.

The data were acquired during two observing runs: February 14-22 2012 and June 6-14 2012. There were no significant changes to the instrument over the course of the project. The conditions during the observations ranged from excellent $\tau_{225 \mathrm{GHz}}=0.03$ to marginal $\tau_{225 \mathrm{GHz}}=0.07$. The observing sequence consisted of a series of target scans bracketed by scans of planets and nearby secondary calibrators, serving as both 

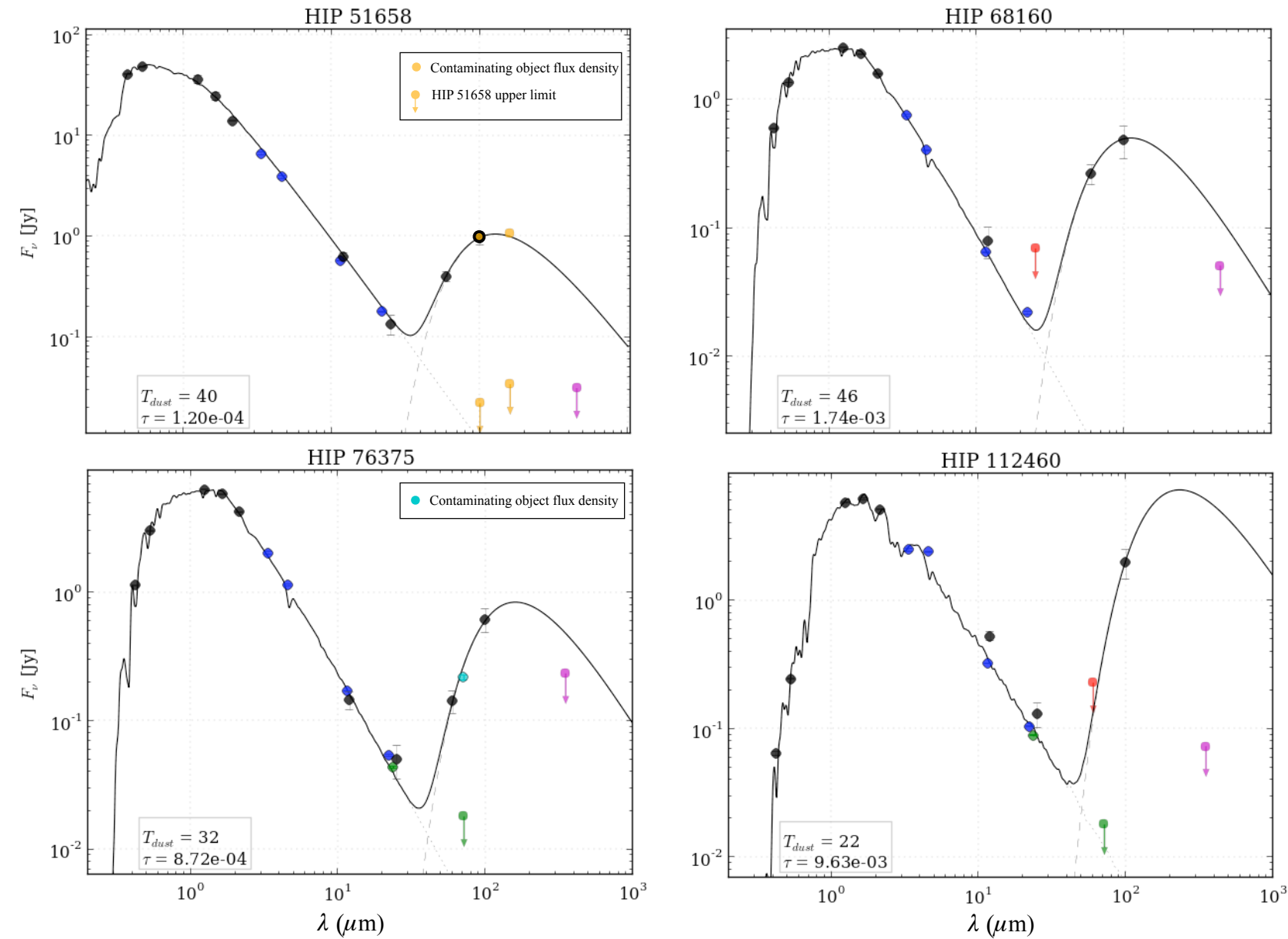

Fig. 1. SEDs of the cold debris disk source candidates for which the submm flux limits are substantially below the extension of the single temperature blackbody fit to the IRAS excesses - HIP 51658, HIP 68160, HIP 76375 and HIP 112460 . The NextGen atmospheric models corresponding to the best-fit stellar photosphere and the blackbody fit are plotted. The optical-near IR photometric points (black points) have been compiled from Tycho2 and 2MASS. The mid-IR photometric points have been drawn from WISE (dark blue points). For HIP 51568, with Herschel PACS photometry, the orange points with downward arrows correspond to the source $3 \sigma$ upper limits. The orange points represent the cumulative flux density of three nearby contaminating objects. For HIP 76375 and HIP 112460, with Spitzer MIPS photometry, the green points represent the source flux density and green points with downward arrows represent the source $3 \sigma$ upper limits. The flux density of the contaminating object measured in the MIPS $70 \mu \mathrm{m}$ image for HIP 76375 is represented by the light-blue point. The CSO submm flux upper limit at 350 or $450 \mu \mathrm{m}$ are represented by the pink points with downward arrows.

Table 2. Submillimeter observations.

\begin{tabular}{lcccccc}
\hline \hline Name & UT date & $\lambda(\mu \mathrm{m})$ & Exp. time & $\tau_{225 \mathrm{GHz}}$ range & Secondary calibrators & Flux calibrators \\
\hline HIP 51658 & 20120216 & 450 & $6 \times 10 \mathrm{~min}$ & $0.04-0.06$ & 3C 273, OH 231.8, OJ 287 & CRL 618, OH 231.8 \\
HIP 68160 & 20120216 & 450 & $5 \times 10 \mathrm{~min}$ & $0.03-0.06$ & 3C 273, OJ 287 & CRL 618, OH 231.8 \\
HIP 73165 & 20120610 & 350 & $5 \times 10 \mathrm{~min}$ & $0.06-0.07$ & Arp 220 & Neptune, Uranus \\
HIP 73512 & 20120610 & 450 & $6 \times 10 \mathrm{~min}$ & $0.06-0.07$ & Arp 220 & Arp 220, Mars \\
HIP 76375 & 20120610 & 350 & $8 \times 5 \mathrm{~min}$ & $0.05-0.06$ & Arp 220 & Neptune, Uranus \\
HIP 112460 & 20120613 & 350 & $8 \times 10 \mathrm{~min}$ & $0.06-0.07$ & Arp 220 & Neptune, Uranus \\
\hline
\end{tabular}

absolute flux calibration and pointing calibration measurements. Table 2 lists the calibrators observed for each science target. Pointing calibration measurements were taken at least once per hour during the observing period and focus corrections were monitored throughout the night. Typical individual target scan times were $620 \mathrm{~s}$, while $120 \mathrm{~s}$ was sufficient for the calibrators. A total of 1 to 11 scans were taken on the science targets.

\subsection{Imaging observations}

High angular resolution observations were used to search for the presence of a stellar binary companion to each member of the sample, since a companion can impact the stability of debris disk material. Dedicated observations were obtained for four targets with the Arizona Infrared Imager and Echelle Spectrograph (ARIES; McCarthy et al. 1998) at the $6.5 \mathrm{~m}$ AO-equipped MMT on December 23, 2012. The observing strategy was the same for each target, with a series of short unsaturated exposures taken first to calibrate the photometry of the observing sequence, followed by deep exposures in which the core of the PSF of the target was saturated, increasing the sensitivity to faint stellar companions. For both sets of exposures, images were obtained at a series of offset positions to measure the sky background and mitigate the effects of bad pixels. Additionally, the CFHT and 
Table 3. High resolution imaging observations.

\begin{tabular}{lcccccccc}
\hline \hline \multicolumn{3}{c}{ This work } & \multicolumn{3}{c}{ Archival data } \\
\hline Name & UT date & Filter & $\begin{array}{c}\text { Instrument/ } \\
\text { telescope }\end{array}$ & $\begin{array}{c}\text { Exp. time } \\
(\mathrm{s})\end{array}$ & UT date & Filter & $\begin{array}{c}\text { Instrument/ } \\
\text { telescope }\end{array}$ & $\begin{array}{c}\text { Exp. time } \\
\text { (s) }\end{array}$ \\
\hline HIP 51658 & 20121223 & $K_{\mathrm{s}}$ & ARIES/MMT & 600 & 20100204 & $K_{\mathrm{p}}$ & AOBIR/CFHT & 440 \\
HIP 68160 & 20121223 & $K_{\mathrm{s}}$ & ARIES/MMT & 620 & 20080321 & $F 606 W$ & WFPC2/HST & 500 \\
HIP 73165 & - & - & - & - & 20050815 & $F 160 W$ & NICMOS/HST & 1663 \\
HIP 73512 & 20121223 & $K_{\mathrm{s}}$ & ARIES/MMT & 450 & 20080309 & $F 160 W$ & NICMOS/HST & 1120 \\
HIP 76375 & - & - & - & - & 20080313 & $F 160 W$ & NICMOS/HST & 1120 \\
HIP 112460 & - & - & - & - & 20020724 & FeII & AOBIR/CFHT & 64 \\
\hline
\end{tabular}

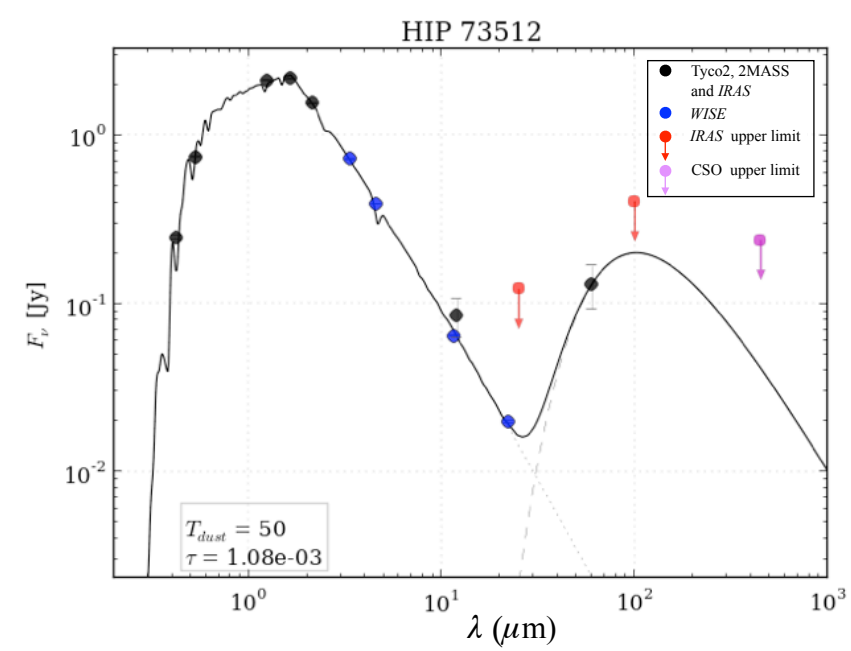

Fig. 2. SED of the cold debris disk candidate - HIP 73512 for which the submm flux limit lies above the extension of the single temperature blackbody fit to the IRAS excesses. The NextGen atmospheric model corresponding to the best-fit stellar photosphere and the blackbody fit are plotted. Deeper submm observations for are required to further assess this source as a cold debris disk candidate.

HST archives were searched for previous high angular resolution observations of each sample member. Collectively, the new and archival observations include all the targets. Details of the high angular resolution observations of the sample are given in Table 3.

To increase the range of separations at which a stellar binary companion can be detected to a limit of $10000 \mathrm{AU}$, digitized photographic plates were obtained from the SuperCosmos Sky Survey Science Archive (Hambly et al. 2001) for each target within the sample. For the targets within $30 \mathrm{pc}$, several of the $15^{\prime} \times 15^{\prime}$ digitized plates were combined to ensure the angular coverage was sufficient to detect co-moving companions with separations up to the 10000 AU outer boundary considered for this study. The large time baseline between the first and last photographic observation, combined with the high proper motion of the sample members, allowed for an identification of wide common proper motion (CPM) companions. An example of one of the wide-field images is shown in Fig. 4.

\subsection{Archival mid-IR/far-IR images}

For each target, images from the all-sky surveys of IRAS and WISE were obtained for this program. Figure 5 shows the region centered on each target for each of the four bands of WISE (left) and four bands of IRAS (right). Overplotted on each image is the approximate size of the $100 \mu \mathrm{m}$ IRAS beam; the beam

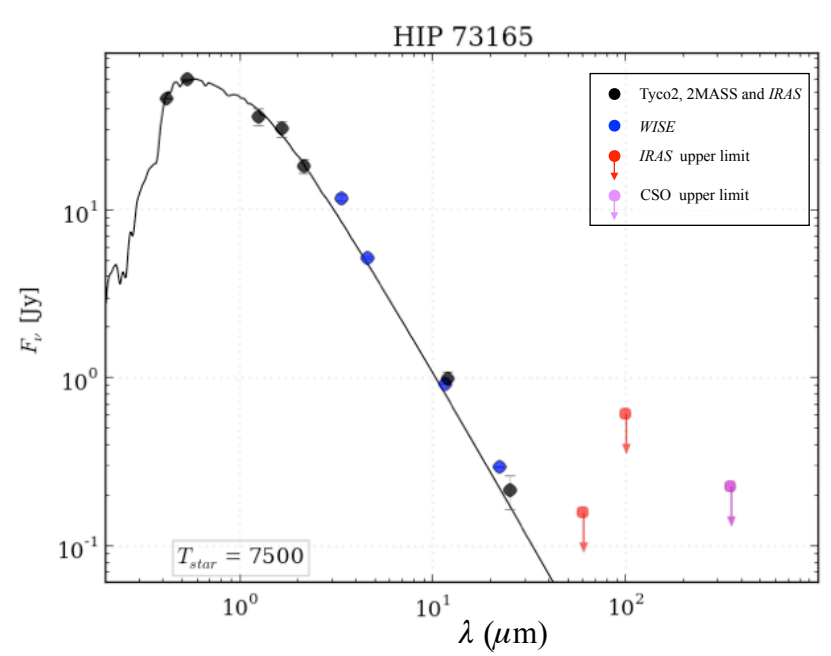

Fig. 3. SED of our WISE excess source - HIP 73165. The NextGen atmospheric model corresponding to the best-fit stellar photosphere is plotted. Excess is seen in the W4 band above the photosphere, and is 34 times the uncertainty in the photometry. The CSO $450 \mu \mathrm{m} 3 \sigma$ upper limit (pink point and downwards arrow) shows the level to which lack of submm excess emission is seen.

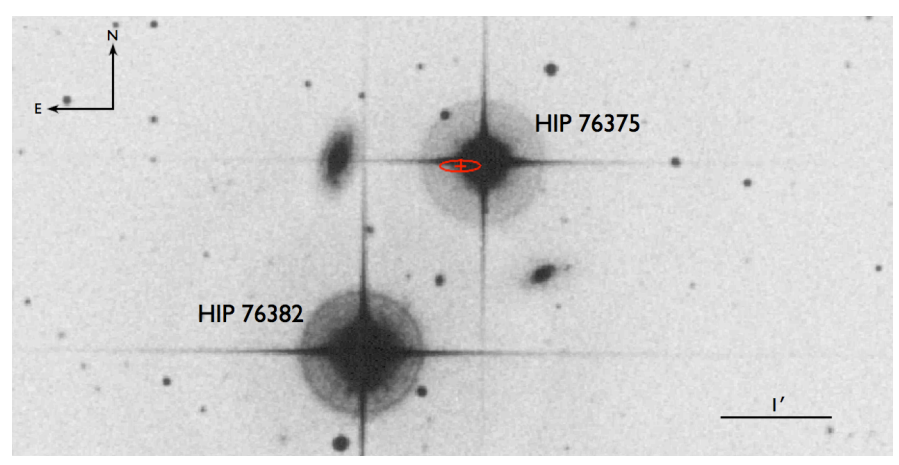

Fig. 4. Field plate image of HIP 76375, the only source in our sample with a known companion. The orientation is indicated with the arrows, along with a scale bar for this $8^{\prime} \times 4^{\prime}$ image. The companion HIP 76382, identified from CPM (Shaya \& Olling 2011) is labeled on the image and lies $148^{\circ}$ from the primary, with a separation of $121^{\prime \prime} 8$. The IRAS FSC positional uncertainty for HIP 76375 is displayed on the image (red ellipse).

is estimated as circular using the average of the elliptical major and minor axis as the radius. We searched the Spitzer Heritage Archive and the Herschel Science Archive for higher resolution, pointed far-IR observations for each target, and three of the targets have additional higher resolution data. Level 2 MIPS data at $24 \mu \mathrm{m}$ and $70 \mu \mathrm{m}$ were retrieved for HIP 76375 and HIP 112460 , and level 2.5 PACS data at 100 and $160 \mu \mathrm{m}$ was retrieved for 

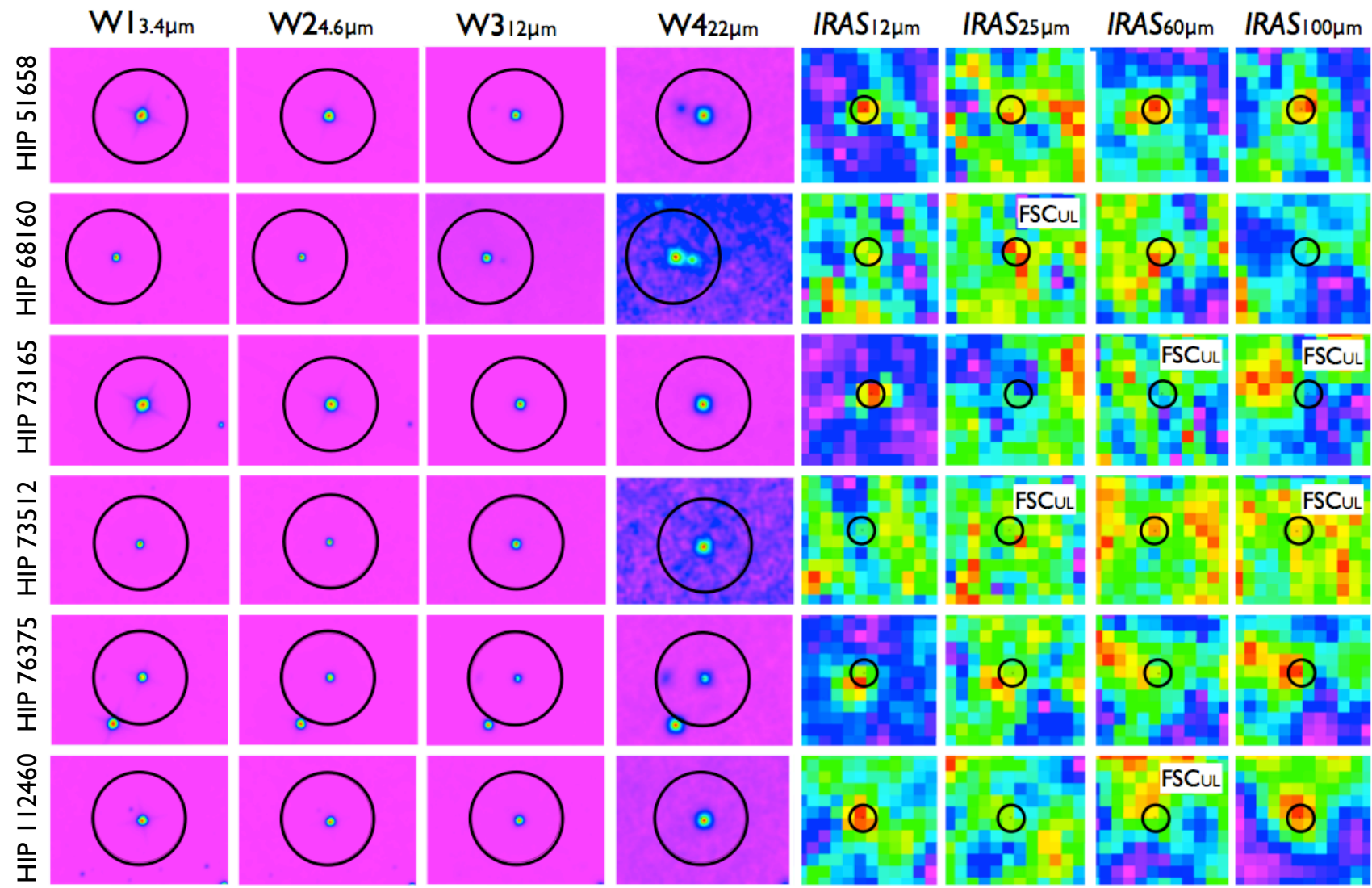

Fig. 5. The WISE All-Sky Survey Atlas images and IRAS Sky Survey Atlas (ISSA) for all the sources of our sample. The images are displayed per source (repented by the row heading) in order of survey, and the wavelength of observation from left to right (indicated by the column heading). Orientation for all images are north, up and east, left. The WISE images are $400^{\prime \prime} \times 300^{\prime \prime}$ in size. The spatial resolution of the ISSA images at each wavelength are smoothed to the IRAS $100 \mu \mathrm{m}$ beam size and are $900^{\prime \prime} \times 900^{\prime \prime}$ in size. The black circles represent the IRAS $100 \mu \mathrm{m}$ beam centered over the source positions. The sources and the wavelengths for which IRAS FSC upper limits are reported are indicted in the images. The four cold debris disks candidates with submm limits well below the SED fit are HIP 51658, HIP 61680 and HIP 76357 and HIP 112460 , and are investigated for the possibility of contaminating sources within the IRAS beam. For HIP 51658, HIP 61680 and HIP 76357 nearby objects are visible in the higher resolution WISE images (see also Fig. 6). Additionally, for HIP 76357, the secondary companion (HIP 76382) is seen dominant in all WISE images and is noticeably seen in the $12 \mu \mathrm{m}$ and $25 \mu \mathrm{m}$ ISSA images. No contaminating sources are visible in either the WISE or ISSA images for HIP 112460.

HIP 51658. The pointed observations with Spitzer and Herschel are shown in Fig. 6.

\section{Data analysis}

\subsection{Submm maps and fluxes}

The analysis of the CSO $350 \mu \mathrm{m}$ and $450 \mu \mathrm{m}$ data included four main steps: application of a pointing model $^{1}$, reduction of the raw data with the CRUSH pipeline (Kovács 2006, 2008), measurement of aperture photometry, and calibration of fluxes. As an initial step, the pointing drifts of the telescope were estimated for each observation with a pointing model that accounts for both static effects, measured with many pointing sources at different positions throughout the run, and for time variable effects, measured with pointing sources observed before and after the science target. These offsets and the contemporaneous measurement of the atmospheric opacity are then incorporated into the data processing, implemented with the CRUSH (version 2.11-2) software (Kovács 2006, 2008). The output of CRUSH includes an intensity map and signal-to-noise ratio map. The image processing algorithm is optimized depending on source brightness.

\footnotetext{
http://www. submm. caltech.edu/ sharc/analysis/pmodel
}

We utilized the data reduction settings appropriate to the flux levels for the planets, pointing sources and science targets. Circular apertures with a radius of 9.'5 at $350 \mu \mathrm{m}$ and $10^{\prime} 6$ at $450 \mu \mathrm{m}$ were used for the target photometry; the aperture sizes are based on the measured FWHM of the point source calibrator at each wavelength.

Absolute fluxes of the planets are based on the distance from the Earth and the Sun, calculated at the time of observation and determined using the planetary brightness temperatures measured by Griffin \& Orton (1993). The planets Mars, Neptune and Uranus were used for primary flux calibration and have absolute calibration uncertainties of $\sim 5-10 \%$ (Griffin \& Orton 1993). For nights without a planet observation, the calibration is tied to the secondary calibrators Arp 220, CRL 618 and OH 231.8. For observations at $350 \mu \mathrm{m}$, we use a flux density of $23.3 \pm 4.2 \mathrm{Jy}$ for CRL 618 (derived by Dowell and listed on the SHARCII website $^{2}$ ). The uncertainty is based on the long term variability of evolved stars (Sandell 2003; Jenness et al. 2002). At $450 \mu \mathrm{m}$, we use a flux density of $6.3 \pm 0.8 \mathrm{mJy}$ for Arp220 (Dunne \& Eales 2001), $12.1 \pm 2.2 \mathrm{mJy}$ for CLR 618 and $12.7 \pm 2.2 \mathrm{mJy}$ for $\mathrm{OH} 231.8$ (Jenness et al. 2002). The uncertainty of the flux calibration is combined with the uncertainty of the source signal

2 http://www. submm. caltech.edu/ sharc/ 

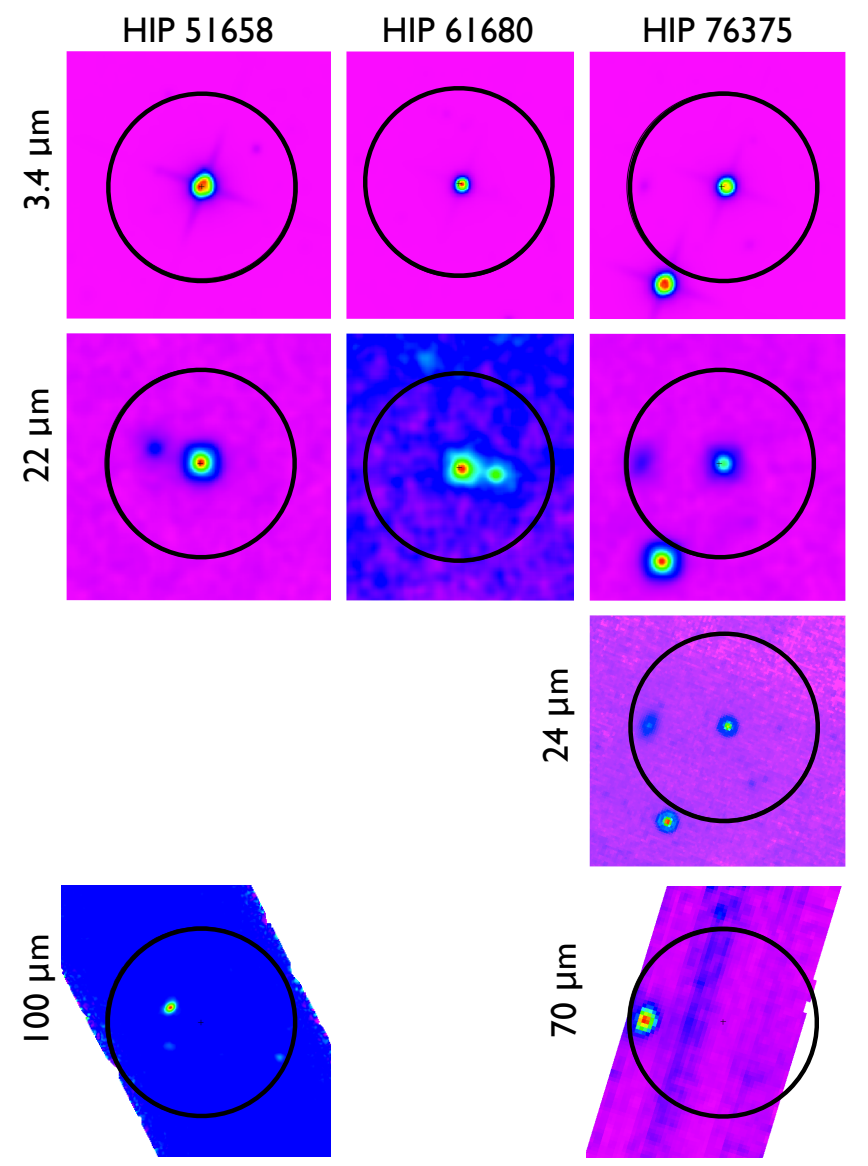

Fig. 6. Higher resolution IR images for the sources - HIP 51568, HIP 61680 and HIP 76375, in which nearby contaminating objects are seen in the WISE and when available Spitzer MIPS and Herschel PACS images. The orientation for all images are north, up and east, left and the images are $300^{\prime \prime}$ in size along each axis. The black circles represent the IRAS $100 \mu \mathrm{m}$ beam centered over the source positions. For HIP 51658 a nearby object ( $\sim 55^{\prime \prime}$ to the NE) is seen to become increasingly brighter at increasing wavelengths, as shown in the WISE $22 \mu \mathrm{m}$ (W4) and Herschel PACS $100 \mu \mathrm{m}$ images. For HIP 68160, a nearby object ( $\sim 35^{\prime \prime}$ to the west) is seen in the W4 image. For HIP 76375 and the binary companion - HIP $76382\left(121^{\prime \prime}\right.$ to the SE) are both visible in the WISE $3.4 \mu \mathrm{m}$ (W1), W4 and Spitzer MIPS $24 \mu \mathrm{m}$ images. Neither component of the system are seen in the Spitzer MIPS $70 \mu \mathrm{m}$ image. The galaxy IC 4563 ( $\sim 80^{\prime \prime}$ to the east), is visible in the W4 and MIPS images, and lies within the IRAS beam area.

which is measured from the CRUSH source maps. The total uncertainty on the submm fluxes of the science targets ranges from $\sim 15-30 \%$.

None of the six targets were detected in the CSO $350 \mu \mathrm{m}$ or $450 \mu \mathrm{m}$ maps. We have therefore pursued two types of analysis in order to investigate the causes for these null detections, presented in Sect. 5. With infrared imaging, we investigate the possible disruptive effects caused by companion stars and with an analysis of available mid-IR to far-IR maps, we explore the possibility of source contamination in the IRAS catalogue resulting in a misidentification of the target as a debris disk system.

\subsection{High resolution and wide-field, near-IR/optical companion search images}

For the ground-based AO images from the MMT and CFHT, we performed standard image processing steps to account for sky background and detector dark current, nonlinearity, bad pixels, and quantum efficiency variations. The individual science images were aligned, and median combined. For the HST archive data, the images were already reduced and flux calibrated, so no additional processing was required. Each final target image was searched for stellar companions, and detection limits were calculated as a function of separation. For each target, the minimum separation at which a stellar companion at the bottom of the main sequence $\left(0.08 M_{\odot}\right)$ could be detected was determined. The variance of the pixel values within the science images was measured as a function of separation, scaled by the flux and apparent magnitude of the central target, and compared to the expected apparent magnitude of a $0.08 M_{\odot}$ stellar companion obtained from theoretical models (Baraffe et al. 1998).

Three of the sample members have also been searched for binary companions with speckle interferometry (HIP 51658, HIP 76375, and HIP 112460; Miura et al. 1995; Hartkopf \& Mason 2009; Balega et al. 2007), and none of which were found to have a binary companion. While these techniques would be sensitive to a high mass ratio binary companion at close separations, their sensitivity does not extend to the bottom of the main sequence. Future observations with increased sensitivity are required in order to rule out the presence of any stellar companion interior to the detection limits given in Table 5 .

To search for companions beyond the field-of-view of the high angular resolution instruments, the digitized photographic plates for each target were blinked to reveal the presence of any wide co-moving companions. To ensure wide CPM companions were not missed during the visual inspection, the PPMXL (Roeser et al. 2010) and the UCAC4 (Zacharias et al. 2012) catalogues were also searched for stars with proper motions consistent with the object being physically bound to the primary.

\subsection{IRAS data}

We visually inspected each image in order to assess the quality and association of IRAS Faint Source Catalogue fluxes that have been extracted for the targets. The synthesized beam sizes of the Faint Source Survey images are approximately $1^{\prime} \times 4^{\prime}$ at $12 \mu \mathrm{m}$ and $25 \mu \mathrm{m}, 2^{\prime} \times 4^{\prime}$ and $4^{\prime} \times 5^{\prime}$ at $60 \mu \mathrm{m}$ and $100 \mu \mathrm{m}$, respectively. The positional uncertainties of IRAS detections for our sample is $\sim 3^{\prime \prime}$ in the in-scan direction and $\sim 18^{\prime \prime}$ in the cross-scan direction. In addition, we have reassessed the quality of the FSC extracted flux densities for our targets, with the use of the online Scan Processing and Integration (SCANPI) tool ${ }^{3}$. SCANPI combines the signal for all (in-scan) survey scans that correspond to the target position and provides fluxes of faint sources, estimates of true local upper limits and deviation of the signal peak to that of the source position. Despite the stringent processing of the survey scans and catalog criteria that have been implemented for source extraction and identification, the large angular resolution of the IRAS beams is one of the primary factors of possible source contamination.

\subsection{Higher resolution mid-far IR WISE, Spitzer and Herschel images}

WISE provides a factor of 10 higher resolution images in the mid-IR than IRAS. The WISE beam sizes are 6"' 1, 6"' 4, 6". 5 and $12^{\prime \prime}$ at $3.4 \mu \mathrm{m}$ (W1), $4.6 \mu \mathrm{m}$ (W2), $12 \mu \mathrm{m}$ (W3) and $22 \mu \mathrm{m}$ (W4). To utilize this significant improvement of resolution, we performed visual cross inspection of the four WISE bands, to

3 http://irsa.ipac.caltech.edu/applications/Scanpi/ 
Table 4. Submillimeter detection limits.

\begin{tabular}{lcccc}
\hline \hline Target & $\begin{array}{c}\lambda \\
(\mu \mathrm{m})\end{array}$ & $\begin{array}{c}\text { Map noise rms } \\
(\mathrm{mJy} / \mathrm{beam})\end{array}$ & $\begin{array}{c}\text { Flux limit }^{\mathrm{a}} \\
(\mathrm{mJy})\end{array}$ & $\begin{array}{c}\text { Dust mass limit } \\
\left(M_{\oplus}\right)\end{array}$ \\
\hline HIP 51658 & 450 & 8 & $<31$ & $<0.046$ \\
HIP 68160 & 450 & 13 & $<51$ & $<0.089$ \\
HIP 73165 & 350 & 66 & $<227$ & $<0.094$ \\
HIP 73512 & 450 & 66 & $<239$ & $<0.262$ \\
HIP 76375 & 350 & 68 & $<234$ & $<0.065$ \\
HIP 112460 & 350 & 22 & $<73$ & $<0.001$ \\
\hline
\end{tabular}

Notes. ${ }^{(a)}$ Flux calibration uncertainties are included in the $3 \sigma$ limits reported. ${ }^{(b)}$ Calculated assuming an average dust temperature of $30 \mathrm{~K}$.

identify any contaminating objects that are contained within the IRAS beam sizes. Additionally, we investigated the available Spitzer MIPS and Herschel PACS (Poglitsch et al. 2010) processed images from the archive for the targets HIP 76375, HIP 112460, and HIP 51658. HIP 68160 was not observed by either Spitzer or Herschel. The Spitzer FWHM beam size with MIPS at $24 \mu \mathrm{m}$ is $6^{\prime \prime}$, a factor two greater than the WISE W4 band, and pointed observations further increase the sensitivity beyond that achieved with the WISE survey. The far-IR MIPS channels at $70 \mu \mathrm{m}$ and $160 \mu \mathrm{m}$, with corresponding beam sizes of $20^{\prime \prime}$ and 40", and the Herschel PACS channels at $70 \mu \mathrm{m}, 100 \mu \mathrm{m}$ and $160 \mu \mathrm{m}$, with FWHM beam sizes of 5.'6, 6.' 8 and $11^{\prime \prime} .4$ respectively, provide the far-IR wavelength coverage with significantly greater resolution than that of IRAS, enabling the identification of contaminating objects such as background galaxies that become increasingly brighter across the mid-far IR regime.

\section{Results}

None of the six debris disk targets were detected in the CSO SHARCII maps. The $3 \sigma$ upper limits range from $31 \mathrm{mJy}$ for HIP 51658 to $239 \mathrm{mJy}$ for HIP 73512 at $450 \mu \mathrm{m}$, and all the limits are reported in Table 4. For four of the five cold debris disks targets, the submm flux limits are substantially below the extension of the blackbody fit to the IRAS excesses, as shown in Fig. 1. The CSO limit for the cold debris disk candidate HIP 73512 is above the blackbody fit, as shown in Fig. 2, and the CSO data do not constrain the disk in this case. The WISE excess source HIP 73165 also shows a lack of submm emission at a level reported in Table 4 and plotted on the SED in Fig. 3.

\subsection{CSO flux limits}

The flux limits were converted into dust mass limits following previous submm studies (e.g. Zuckerman \& Becklin 1993) using the expression $M_{\mathrm{d}}=\left(F_{v} D^{2}\right) / B_{v}(T) \kappa_{v}$, where the blackbody function $B_{v}(T)$ is equal to $2 k T / \lambda^{2}$ in the Rayleigh-Jeans limit. The dust opacity at the frequency of observation $\kappa_{v}$ assumes a functional form $\kappa_{v}=\kappa_{0}\left(v / v_{0}\right)^{\beta}$ at submm wavelengths. We use a normalization value $\kappa_{0}=0.17 \mathrm{~m}^{2} \mathrm{~kg}^{-1}$ at $850 \mu \mathrm{m}$ and scale with $\beta=1$ (Zuckerman \& Becklin 1993; Pollack et al. 1994). Existing submm studies find that $\beta=1$ is a representative value of debris disks (Dent et al. 2000) and the normalization value for dust opacity is adopted for direct comparison with previous studies (e.g. Sylvester et al. 2001; Wyatt et al. 2003; Liu et al. 2004; Sheret et al. 2004; Najita \& Williams 2005; Williams \& Andrews 2006). Assuming an average dust temperature of $30 \mathrm{~K}$ for the submm-emitting dust, the submm flux limits correspond to dust mass limits of 0.001 to $0.261 M_{\oplus}$, as reported in Table 4 .
These dust mass limits are comparable to the detections of other nearby debris disks based on either submm fluxes or fits to IRAS fluxes over a large wavelength range (e.g. compilation in Rhee et al. 2007).

The submm upper limits are plotted along with previous measurements at shorter wavelengths to construct the SEDs of the IRAS cold excess debris disks with limits below the blackbody fit in Fig. 1 and with a limit above the blackbody fit in Fig. 2. The WISE warm excess debris disk SED is plotted in Fig. 3. For the cold excess sources, the fits to the $60-100 \mu \mathrm{m}$ emission, assuming a single temperature blackbody, are also shown for comparison with the measured CSO limits. For the targets HIP 51658, HIP 68160, HIP 76375 and HIP 112460, the submm limits are all well below the expected flux level at these wavelengths, making these limits more restrictive than previous submm measurements of some debris disks candidates Williams \& Andrews (2006). For the WISE source, there is not a large enough wavelength coverage for the excess emission to perform a fit, and the exploratory measurement at the longer wavelength is shown in comparison to the newly reported mid-IR excess in the WISE W4 band. With only an excess in the longest wavelength WISE band, it is not possible to fit a blackbody to estimate an expected flux level for the CSO measurement, but the non-detection is consistent with a spatially confined distribution of hot dust for this source. Larger scale studies with more sensitive limits from Herschel will provide more definitive constraints on the origin of WISE excesses for sources such as HIP 73165.

\subsection{Companion systems}

Since a companion star will gravitationally truncate the outer portion of a disk and reduce the amount of submm-emitting dust, all targets were searched for stellar companions. A visual inspection of the high-resolution data did not reveal any companion candidates. Separately, a visual inspection of each of the blinked image pairs combined with a search of the astrometric catalogues, identified one co-moving object - the known wide CPM companion to HIP 76375 (Shaya \& Olling 2011). The HIP 76375 system is shown in Fig. 4. The binary separation is 121 .' 8 , which corresponds to a projected physical separation of $\sim 2700 \mathrm{AU}-$ too large to impact the disk size unless the orbit is very eccentric and periastron passage occurs at a much smaller separation. Table 5 lists the details of the HIP 76375 binary system and the closest separation at which the data become sensitive to companions at the bottom of the main sequence for each of the targets. The minimum separation ranges from only $1 \mathrm{AU}$ for the M-star HIP 112460 at $\sim 5$ pc to 94 AU for the more massive and more distant A-star HIP 51658 at $\sim 30$ pc. In addition to the the companion search we performed, five targets were included in the interferometric (van Belle et al. 2008; van Belle 2010) and speckle (Hartkopf \& McAlister 1984; Miura et al. 1995; Hartkopf \& Mason 2009; Balega et al. 2007) searches, and no companions were discovered for these targets, although the detection limits were not deep enough to include all possible stellar companions.

\subsection{IRAS source contamination}

For the four cold debris disk candidates with submm limits well below the SED fit (shown in Fig. 1), we investigated the possibility of contaminating sources within the IRAS beam. All targets have WISE images in four bands with higher resolution than IRAS, and three of the four targets have Spitzer data in 
Table 5. Companion search results.

\begin{tabular}{lcccccc}
\hline \hline Target & $\begin{array}{c}\text { Radius to reach bottom of MS } \\
(\mathrm{AU})\end{array}$ & $\begin{array}{c}\text { Detected } \\
\text { companion }\end{array}$ & $\begin{array}{c}\text { Companion separation } \\
(\mathrm{AU})\end{array}$ & $\begin{array}{c}\text { Position angle } \\
\left({ }^{\circ}\right)\end{array}$ & $\begin{array}{c}\Delta \text { mag } \\
(\mathrm{mag})\end{array}$ & $\begin{array}{c}\text { Technique } \\
\text { HIP 51658 }\end{array}$ \\
\hline 39 & $\mathrm{~N}$ & - & - & - & - \\
HIP 68160 & 94 & $\mathrm{~N}$ & - & - & - & - \\
HIP 73165 & 45 & $\mathrm{~N}$ & - & - & - & - \\
HIP 73512 & 41 & $\mathrm{~N}$ & - & - & - & - \\
HIP 76375 & 35 & $\mathrm{Y}$ & 2725 & - & - & CPM \\
HIP 112460 & 1 & $\mathrm{~N}$ & - & - \\
\hline
\end{tabular}

at least one bandpass. Three of the four objects - HIP 51658, HIP 61680, and HIP 76375 - show contaminating sources in the higher resolution mid-IR/far-IR images shown in Fig. 6. Only one - HIP 112460 - shows a single object in the higher resolution WISE and Spitzer maps shown in Fig. 7. The results of the contamination analysis for these sources is discussed in the following subsections.

\subsubsection{HIP 51658}

From the sequence of WISE images for HIP 51658 in Figs. 5 and 6, a nearby object $\sim 55^{\prime \prime}$ to the NE of HIP 51658 is seen and becomes increasingly brighter with wavelength. Also shown in Fig. 6 is the Herschel PACS $100 \mu \mathrm{m}$ map centered on the target which reveals the offset source, but not the target. In both the WISE and Herschel maps there are additional fainter sources within the IRAS beam (not visible with the scaling of Fig. 6). Three distinct sources are detected in the Herschel $100 \mu \mathrm{m}$ map. From our SCANPI analysis of this source, we find that signals are significant in all IRAS bands $(>5 \sigma)$ and the the deviation from source position to the signal peak is $<0.5^{\prime}$.

To determine the flux of the contaminating objects, we have measured the flux densities of the nearby three objects that fall within the IRAS beam centered over the source position, and find that the cumulative flux density at $100 \mu \mathrm{m}$ is consistent with the IRAS FSC $100 \mu \mathrm{m}$ flux. Furthermore, the $160 \mu \mathrm{m}$ cumulative flux density fits well to the single temperature blackbody, modelled excess fit. The PACS fluxes, along with our $450 \mu \mathrm{m}$ upper limit that lies significantly below the fit, are shown in Fig. 1. This analysis indicates that the associated IRAS $60 \mu \mathrm{m}$ and $100 \mu \mathrm{m}$ fluxes of this source are contaminated and that HIP 51658 is not a bona fide debris disk system. To determine more accurate flux upper limits for HIP 51658, we have extracted level 2.5 processed PACS $100 \mu \mathrm{m}$ and $160 \mu \mathrm{m}$ images from the Herschel archive and measured $3 \sigma$ flux upper limits of $22 \mathrm{mJy}$ at $100 \mu \mathrm{m}$ and $34 \mathrm{mJy}$ at $160 \mu \mathrm{m}$, following the point source photometry guidelines recommended by the Herschel team ${ }^{4}$.

\subsubsection{HIP 68160}

As shown in Fig. 6, a nearby object $\sim 35^{\prime \prime}$ to the west of HIP 68160 is visible in the W4 $22 \mu \mathrm{m}$ image, and lies within the IRAS beam. The IRAS $25 \mu \mathrm{m}$ flux for this source is reported as an upper limit in the FSC. As there are no higher resolution far-IR observations available from Spitzer or Herschel for this source, we are unable to quantify the level of flux contamination within the IRAS $60 \mu \mathrm{m}$ and $100 \mu \mathrm{m}$ bands, unlike the case of HIP 51658. The presence of the additional source in the WISE W4 image does, however, suggest that the IRAS fluxes

\footnotetext{
4 Technical Note PICC-ME-TN-037 in http://herschel.esac. esa.int
}

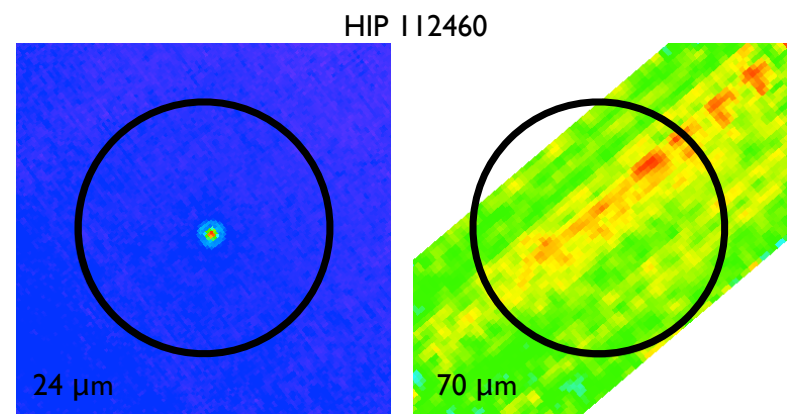

Fig. 7. Higher resolution Spitzer MIPS images for HIP 112460. The orientation of both images are north, up and east, left and the images are $300^{\prime \prime}$ in size along each axis. The black circles represent the IRAS $100 \mu \mathrm{m}$ beam centered over the source position. The source is clearly detected in the $24 \mu \mathrm{m}$ image, with no visible nearby contaminating objects. The $3 \sigma$ upper limit of the source measured in the $70 \mu \mathrm{m}$ image lies well below the modelled, single blackbody excess fit. Whilst stripping artifacts are seen in the $70 \mu \mathrm{m}$ image no nearby contaminating objects are visible.

are heavily influenced by this red object. Based on this second source in the field, HIP 68160 is also a probable case of a misclassified debris disk system.

\subsubsection{HIP 76375}

This target is the only known binary in our sample. Both the target and binary companion (separation of 121'.8) are visible in all four WISE images (see Fig. 5) and the MIPS $24 \mu \mathrm{m}$ image (see Fig. 6). From our SCANPI analysis we note that there is a large positional offset $\left(1.9^{\prime}\right)$ from the source to signal peak in the IRAS $12 \mu \mathrm{m}$ band, with a SNR of 8 . Figure 5 shows the IRAS scans at $12 \mu \mathrm{m}, 60 \mu \mathrm{m}$ and $100 \mu \mathrm{m}$. In the $12 \mu \mathrm{m}$ scan, the position of the binary companion falls on the peak emission pixel, indicating that the IRAS $12 \mu \mathrm{m}$ flux reported for this source contaminated is from emission of the secondary.

To investigate the longer wavelength measurements, we have extracted the archival MIPS images at $24 \mu \mathrm{m}$ and $70 \mu \mathrm{m}$ for this source, shown in Fig. 6. In all the WISE images and in the MIPS $24 \mu \mathrm{m}$ image, both the target and companion are visible. The galaxy, IC $45634^{5}\left(\sim 80^{\prime \prime}\right.$ to the E) is seen to become increasingly brighter with longer wavelengths, as seen in Figs. 5 and 6. In the MIPS $70 \mu \mathrm{m}$ image, neither the source or companion are visible, yet the galaxy is clearly seen and lies within the IRAS $100 \mu \mathrm{m}$ beam, centered over the source position. The SNR of the signal, returned with SCANPI at $60 \mu \mathrm{m}$ is marginal at $2.9 \sigma$ and the positional offset from the source to signal peak is $0.33^{\prime}$.

\footnotetext{
5 Identified in the NASA/IPAC Extragalactic Database - http:// ned.ipac.caltech.edu/
} 
Whilst this offset is below the FSC criteria to flag the source as an upper limit, it is significantly greater than that of other $60 \mu \mathrm{m}$ source detections (as determined through comparison of our sources and those of a similar study i.e. Williams \& Andrews 2006 - as is discussed in Sect. 6).

From the MIPS images we have measured the source flux at $24 \mu \mathrm{m}$, the $3 \sigma$ upper limit at $70 \mu \mathrm{m}$, and the flux of the galaxy (light blue point); included on the SED shown in Fig. 1. We find that the source $24 \mu \mathrm{m}$ flux lies on the modelled photosphere of this object and that the $70 \mu$ m upper limit lies significantly below the single blackbody, model excess fit. Furthermore, we find that the $70 \mu \mathrm{m}$ flux of the galaxy matches well to the excess fit. In visual inspection of the IRAS scans it can be seen that at the position of the galaxy, the corresponding pixel becomes increasingly brighter at longer wavelengths. From this analysis, we conclude that both the IRAS $60 \mu \mathrm{m}$ and $100 \mu \mathrm{m}$ flux is contaminated due to the emission from the galaxy.

\subsubsection{HIP 112460}

Although the HIP 112460 M-star cold debris disk candidate has the largest discrepancy between the predicted submm flux from the SED and the measured CSO limit (a factor of $\sim 100$ ), no additional source was identified within the IRAS beam. From inspection of the higher resolution WISE and MIPS images, no contaminating object is detected in the surrounding field of this source, as shown in Fig. 7. Due to the low latitude of the source $\left(b=-13^{\circ}\right)$, it is possible that the associated $100 \mu \mathrm{m}$ IRAS flux is contaminated from Galactic cirrus emission. Another possibility for this nearby source is a dilution of the flux from a disk due to a spatially resolved disk. To assess the impact of a spatially resolved disk, we searched for the largest reported spatially resolved disks around M-stars (AU Mic - MacGregor et al. 2013, and GJ 581 - Lestrade et al. 2012), scaled the disk size based on the distance to HIP 112460, and determined the number of beams across which the flux would be distributed in a uniform disk. Even for the larger of the two resolved M-star disks, the resulting size at the distance of HIP 112460 would spread the flux across $\sim 9$ beams, diluting the flux by a factor too small to account for the large discrepancy.

Another possible explanation for the submm non-detection is a variable source. Some support for this hypothesis comes from the conflicting flux measurements at different epochs, since the fluxes obtained from later observations with WISE at $12 \mu \mathrm{m}$ and $22 \mu \mathrm{m}$ and Spitzer MIPS at $24 \mu \mathrm{m}$ fall on the photosphere of the star, compared to the excesses obtained from the earlier IRAS at 12 and $25 \mu \mathrm{m}$ scans, and seen at high significance at $12 \mu \mathrm{m}$ and $100 \mu \mathrm{m}$ in Fig. 5. From the Spitzer MIPS images of this source, we measured the $24 \mu \mathrm{m}$ flux and the $70 \mu \mathrm{m}$ upper limit. As shown in Fig. 1, the Spitzer $24 \mu \mathrm{m}$ flux lies on the modelled stellar photosphere for this source and both the $3 \sigma$ upper limits at $70 \mu \mathrm{m}$ and our $350 \mu \mathrm{m}$ observation, lie well below the modelled, single blackbody excess fit (unlike the IRAS values). A large scale IRAS variability investigation of blazars (Impey $\&$ Neugebauer 1988) did find sources with variability up to an order of magnitude at $100 \mu \mathrm{m}$, however such a large amplitude change was very rare. Another example of a large degree of variability intrinsic to a debris disk at somewhat shorter wavelengths in the mid-IR was reported for the TYC 824126521 debris disk (Melis et al. 2012) which exhibited a remarkable change of a factor of $\sim 30$ over a timescale of less than two years. More observations are required to provide further understanding into the nature of this unusual, possibly variable, system.

\section{Investigation of other debris disks with upper limits}

The high rate of source contamination of the IRAS FSC fluxes found amongst our cold debris disk sample prompted us to conduct the same contamination analysis for a previous submm study, at $850 \mu \mathrm{m}$ of eight debris disks (Williams \& Andrews 2006), to further assess the likelihood of additional examples of misidentified debris disks. In the Williams \& Andrews (2006) study, the sources were selected through IRAS FSC excesses, similar to our program. Upper-limits were obtained for two of the eight sources - HD 56099 and HD 78702. From inspection of the higher resolution WISE and Spitzer MIPS images of these two sources, we find that a nearby object lies within the IRAS beam size for HD 56099. No nearby contaminating objects are visible in either the WISE or Spitzer images for HD 78702. For HD 56099, the nearby object is visible in the WISE W3 and W4 images and in the MIPS $24 \mu \mathrm{m}, 70 \mu \mathrm{m}$ and $160 \mu \mathrm{m}$ images, and becomes increasingly brighter with wavelength. Moór et al. (2006) identify this source to be a misclassified debris disk system, due to the nearby object contamination as identified, and Moór et al. (2011) have further analysed the Spitzer observations for this target and find a low significance level of excess above that of the stellar photosphere. One out of eight sources, in the study carried out by Williams \& Andrews (2006), was misidentified of possessing a cold debris disk. Comparatively, for the cold debris disk sample within this paper, three out of the five sources show evidence of nearby object contamination of the associated IRAS FSC fluxes.

\section{Conclusions}

We have presented new submm observations at either $350 \mu \mathrm{m}$ or $450 \mu \mathrm{m}$, searching for dust emission around a sample of cold and warm nearby $(<40 \mathrm{pc})$ debris disk candidates that have been identified from IRAS or WISE excesses.

Upper-limits were obtained for all six targets, and four of the limits are well below the expected submm flux, based on a single temperature blackbody model fit to previous shorter wavelength photometry. Of the four cold excess sources with very discrepant submm limits compared to the SED fit, we found that three targets have clearly identified contaminating objects in the IRAS beam, and, therefore, are likely misidentified debris disk candidates. Quantitive measurements of the contaminating objects were obtained for two targets - HIP 51658 and HIP 76375 - from higher resolution, pointed Herschel PACS and Spitzer MIPS observations at wavelengths comparable to the IRAS excess fluxes. From visual inspection of the WISE all-sky survey images, we find that HIP 61680 is also like to suffer from IRAS contamination, in which we identify a nearby, background object that becomes increasingly brighter over the WISE bands as the likely true source of the IRAS FSC measurement.

The rate of IRAS contamination has likely lead to a greater number of sources in being identified as possessing cold debris disks than the actual number. Considering the high level of source contamination in this study and comparable results from an analysis of other debris disks candidates with submm nondetections (Williams \& Andrews 2006), lower detection rates than anticipated for large scale debris disks surveys that are currently being pursued with Herschel and SCUBA2 may be found.

It is important to highlight the need for careful examination of source contamination for observations in the far-IR and submm wavelengths, where the probability of detecting a nearby 
background source can be as high as 36\% (Booth et al. 2013; Berta et al. 2011). In this study we find 4-5 examples of contamination either from identified objects or possible background variability, giving a contamination rate of $31-38 \%$ similar to the probability reported in Booth et al. (2013).

Finally, we note that it is only now that it is possible to assess systematically the IRAS associations of debris disk candidates by using the complementary mid-IR to far-IR wavelength coverage and higher resolution and sensitivity provided by WISE, Spitzer and Herschel.

Acknowledgements. This material is based upon work at the Caltech Submillimeter Observatory, which is operated by the California Institute of Technology under cooperative agreement with the National Science Foundation (AST-0838261) We gratefully acknowledge support to Exeter from the Leverhulme Trust (F/00144/BJ) and STFC (ST/F0071241/1, ST/H002707/1). This research is partly based on data obtained from the ESO Science Archive Facility, and the CFHT Science Archive. Based on observations made with the NASA/ESA Hubble Space Telescope, obtained from the data archive at the Space Telescope Science Institute. STScI is operated by the Association of Universities for Research in Astronomy, Inc. under NASA contract NAS 5-26555. This research has made use of the SIMBAD database, operated at CDS, Strasbourg, France. This publication makes use of data products from the Two Micron All Sky Survey, which is a joint project of the University of Massachusetts and the Infrared Processing and Analysis Center/California Institute of Technology, funded by the National Aeronautics and Space Administration and the National Science Foundation. This research has made use of the Washington Double Star Catalog maintained at the US Naval Observatory. This research has made use of data obtained from the SuperCOSMOS Science Archive, prepared and hosted by the Wide Field Astronomy Unit, Institute for Astronomy, University of Edinburgh, which is funded by the UK Science and Technology Facilities Council. This research used the facilities of the Canadian Astronomy Data Centre operated by the National Research Council of Canada with the support of the Canadian Space Agency. This research has made use of the NASA/IPAC Infrared Science Archive, which is operated by the Jet Propulsion Laboratory, California Institute of Technology, under contract with the National Aeronautics and Space Administration. This publication makes use of data products from the Wide-field Infrared Survey Explorer, which is a joint project of the University of California, Los Angeles, and the Jet Propulsion Laboratory/California Institute of Technology, funded by the National Aeronautics and Space Administration.

\section{References}

Aumann, H. H., Beichman, C. A., Gillett, F. C., et al. 1984, ApJ, 278, L23 Backman, D. E., \& Paresce, F. 1993, in Protostars and planets III (A93-42937 17-90), 1253

Balega, I. I., Balega, Y. Y., Maksimov, A. F., et al. 2007, Astrophys. Bull., 62, 339

Baraffe, I., Chabrier, G., Allard, F., \& Hauschildt, P. H. 1998, A\&A, 337, 403

Beichman, C. A., Tanner, A., Bryden, G., et al. 2006, ApJ, 639, 1166

Berta, S., Magnelli, B., Nordon, R., et al. 2011, A\&A, 532, A49

Booth, M., Kennedy, G., Sibthorpe, B., et al. 2013, MNRAS, 428, 1263

Bryden, G., Beichman, C. A., Trilling, D. E., et al. 2006, ApJ, 636, 1098

Cameron, A. G. W. 1997, Icarus, 126, 126

Carpenter, J. M., Bouwman, J., Mamajek, E. E., et al. 2009, ApJS, 181, 197

Cutri, R. M., et al. 2012, VizieR On-line Data Catalog, II/311

Dent, W. R. F., Walker, H. J., Holland, W. S., \& Greaves, J. S. 2000, MNRAS, 314,702

Dowell, C. D., Allen, C. A., Babu, R. S., et al. 2003, Millimeter and

Submillimeter Detectors for Astronomy, eds. T. G. Phillips, \& J. Zmuidzinas, 4855,73

Dunne, L., \& Eales, S. A. 2001, MNRAS, 327, 697

Griffin, M. J., \& Orton, G. S. 1993, Icarus, 105, 537

Habing, H. J., Dominik, C., de Muizon, M. J., et al. 1999, Nature, 401, 456
Hambly, N. C., MacGillivray, H. T., Read, M. A., et al. 2001, MNRAS, 326, 1279

Hartkopf, W. I., \& Mason, B. D. 2009, AJ, 138, 813

Hartkopf, W. I., \& McAlister, H. A. 1984, ASP, 96, 105

Hauschildt, P. H., Allard, F., \& Baron, E. 1999, ApJ, 512, 377

Hillenbrand, L. A., Carpenter, J. M., Kim, J. S., et al. 2008, ApJ, 677, 630

Holland, W. S., Greaves, J. S., Zuckerman, B., et al. 1998, Nature, 392, 788

Hughes, A. M., Wilner, D. J., Andrews, S. M., et al. 2011, ApJ, 740, 38

Impey, C. D., \& Neugebauer, G. 1988, AJ, 95, 307

Jayawardhana, R., Holland, W. S., Kalas, P., et al. 2002, ApJ, 570, L93

Jenness, T., Stevens, J. A., Archibald, E. N., et al. 2002, MNRAS, 336, 14

Kovács, A. 2006, Proquest Dissertations And Theses, Section 0036, 28

Kovács, A. 2008, Millimeter and Submillimeter Detectors and Instrumentation for Astronomy IV, eds. W. D. Duncan, W. S. Holland, S. Withington, \& J. Zmuidzinas, 7020, 45

Kuchner, M. J., \& Holman, M. J. 2003, ApJ, 588, 1110

Leong, M., Peng, R., Houde, M., et al. 2006, Millimeter and Submillimeter Detectors and Instrumentation for Astronomy III, eds. J. Zmuidzinas, W. S. Holland, S. Withington, \& W. Duncan, 6275, 21

Lestrade, J.-F., Wyatt, M. C., Bertoldi, F., Dent, W. R. F., \& Menten, K. M. 2006, A\&A, 460, 733

Lestrade, J.-F., Matthews, B. C., Sibthorpe, B., et al. 2012, A\&A, 548, A86

Liou, J.-C., \& Zook, H. A. 1999, AJ, 118, 580

Lisse, C., Schultz, A., Fernandez, Y., et al. 2002, ApJ, 570, 779

Liu, M. C., Matthews, B. C., Williams, J. P., \& Kalas, P. G. 2004, ApJ, 608, 526

MacGregor, M. A., Wilner, D. J., Rosenfeld, K. A., et al. 2013, ApJ, 762, L21

Mannings, V., \& Barlow, M. J. 1998, ApJ, 497, 330

McCarthy, D. W., Ge, J., Hinz, J. L., et al. 1998, Am. Astron. Soc., 193, 1265

Melis, C., Zuckerman, B., Rhee, J. H., et al. 2012, Nature, 487, 74

Miura, N., Iribe, T., Kubo, T., Baba, N., \& Isobe, S. 1995, Publ. Nat. Astron. Obs. Japan, 4, 67

Moór, A., Ábrahám, P., Derekas, A., et al. 2006, ApJ, 644, 525

Moór, A., Pascucci, I., Kóspál, Á., et al. 2011, ApJS, 193, 4

Moshir, M., Kopman, G., \& Conrow, T. A. O. 1992, Pasadena: Infrared Processing and Analysis Center

Najita, J., \& Williams, J. P. 2005, ApJ, 635, 625

Patience, J., Bulger, J., King, R. R., et al. 2011, A\&A, 531, L17

Perryman, M. A. C., \& ESA 1997, The Hipparcos and Tycho catalogues, Astrometric and photometric star catalogues derived from the ESA HIPPARcos Space Astrometry Mission, 1200

Poglitsch, A., Waelkens, C., Geis, N., et al. 2010, A\&A, 518, L2

Pollack, J. B., Hollenbach, D., Beckwith, S., et al. 1994, ApJ, 421, 615

Quillen, A. C., \& Faber, P. 2006, MNRAS, 373, 1245

Rhee, J. H., Song, I., Zuckerman, B., \& McElwain, M. 2007, ApJ, 660, 1556

Rieke, G. H., Su, K. Y. L., Stansberry, J. A., et al. 2005, ApJ, 620, 1010

Roeser, S., Demleitner, M., \& Schilbach, E. 2010, AJ, 139, 2440

Sandell, G. 2003, The calibration legacy of the ISO Mission, 481, 439

Shaya, E. J., \& Olling, R. P. 2011, ApJS, 192, 2

Sheret, I., Dent, W. R. F., \& Wyatt, M. C. 2004, MNRAS, 348, 1282

Silverstone, M. D. 2000, Ph.D. Thesis, University of California

Smith, B. A., \& Terrile, R. J. 1984, Science, 226, 1421

Song, I., Weinberger, A. J., Becklin, E. E., Zuckerman, B., \& Chen, C. 2002, AJ, 124,514

Stern, S. A. 1996, A\&A, 310, 999

Su, K. Y. L., Rieke, G. H., Stansberry, J. A., et al. 2006, ApJ, 653, 675

Sylvester, R. J., Dunkin, S. K., \& Barlow, M. J. 2001, MNRAS, 327, 133

van Belle, G. T. 2010, Rev. Mex. Astron. Astrofís, 38, 119

van Belle, G. T., van Belle, G., Creech-Eakman, M. J., et al. 2008, ApJS, 176, 276

van Leeuwen, F. 2007, A\&A, 474, 653

Williams, J. P., \& Andrews, S. M. 2006, ApJ, 653, 1480

Wyatt, M. C. 2006, ApJ, 639, 1153

Wyatt, M. C. 2008, ARA\&A, 46, 339

Wyatt, M. C., Dent, W. R. F., \& Greaves, J. S. 2003, MNRAS, 342, 876

Zacharias, N., Finch, C. T., Girard, T. M., et al. 2012, VizieR On-line Data Catalog, I/322

Zuckerman, B. 2001, ARA\&A, 39, 549

Zuckerman, B., \& Becklin, E. E. 1993, ApJ, 414, 793 